\title{
A Potential Complication of COVID-19: A Case of Non-Traumatic Rhabdomyolisis
}

\author{
Aakash Sathappan ${ }^{\mathrm{a}, \mathrm{c}}$, David Wachs ${ }^{\mathrm{b}}$, Carrie Morgenstein ${ }^{\mathrm{b}}$
}

\begin{abstract}
Rhabdomyolysis can present as a clinical challenge due to broad etiological spectrum, non-specific symptomatology and numerous systemic complications including acute kidney injury. Emerging evidence suggests that rhabdomyolysis may be an associated early or late complication of coronavirus disease 2019 (COVID-19) infection. We present a case of a 55-year-old woman with recent COVID-19 pneumonia who was later found to have non-traumatic rhabdomyolysis incidentally captured on COVID-19 screening labs.
\end{abstract}

Keywords: COVID-19; Coronavirus; Rhabdomyolysis; SARSCoV-2; Creatine kinase; Acute renal failure

\section{Introduction}

The clinical presentation of coronavirus disease 2019 (COVID-19) is broad, ranging from mild disease, characterized by few or no symptoms, to critical disease marked by respiratory failure, septic shock and/or multi-organ failure [1]. Typical symptoms include fever, cough, dyspnea and sore throat [2]. The literature to date has documented complications from COVID-19 infection affecting the respiratory, cardiac and renal systems [2]. Emerging evidence suggests that the severe acute respiratory syndrome coronavirus 2 (SARS-CoV-2) has potential to affect several other organ systems including the neurologic and musculoskeletal systems $[3,4]$. As such, a high index of suspicion is needed in COVID-19 patients to identify the development of these systemic complications.

One potential complication is rhabdomyolysis which often presents as a clinical challenge due to broad etiological spectrum, non-specific symptomatology and numerous sys-

Manuscript submitted July 16, 2020, accepted July 24, 2020

Published online August 6, 2020

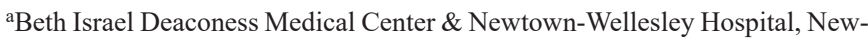
town, MA, USA

bMCPHS University, Boston, MA, USA

${ }^{\mathrm{c} C o r r e s p o n d i n g ~ A u t h o r: ~ A a k a s h ~ S a t h a p p a n, ~ B e t h ~ I s r a e l ~ D e a c o n e s s ~ M e d i c a l ~}$

Center \& Newtown-Wellesley Hospital, Newtown, MA, USA.

Email: asathapp@bidmc.harvard.edu

doi: https://doi.org/10.14740/jmc3546 temic complications [5]. A high degree of clinical suspicion is needed to make the diagnosis as the classic triad of myalgia, myoglobinuria and weakness is seen in less than $10 \%$ of patients and over $50 \%$ of affected patients report neither muscle pain nor weakness [6]. Early detection and treatment is essential to prevent acute kidney injury, one of the more severe complications that develops in nearly one-third of patients as a result of nephrotoxic myoglobin accumulation [7]. We present a case of patient who had recently recovered from COVID-19 pneumonia and was subsequently found to have rhabdomyolysis that we suspect was related to her SARS-CoV-2 infection. Our aim was to inform other clinicians about this rare yet serious complication that has a documented association with this novel coronavirus.

\section{Case Report}

The patient was a 55-year-old female who initially presented to the hospital for hypoxia in the setting of mild COVID-19 infection confirmed via nasopharyngeal polymerase chain reaction (PCR) swab and treated supportively with oxygen and statin medication for immunomodulatory effect. She did not require intubation, high-flow oxygenation, or intensive care unit (ICU) level of care. She was then readmitted approximately 10 days after discharge from the hospital for severe hyperglycemia and acute change in physical mobility. On readmission, screening labs were notable for positive SARS-CoV-2 PCR via nasopharyngeal swab (she had previously tested negative prior to discharge) and elevated creatine kinase (CK). Her past medical history was notable for traumatic fall in childhood complicated by left hemiparesis and cognitive impairment, insulindependent diabetes mellitus, history of deep vein thrombosis on chronic anticoagulation, unspecified seizure disorder on antiepileptics and bipolar disorder on a chronic antipsychotic regimen.

On admission, her vital signs were stable with temperature of $36.7^{\circ} \mathrm{C}$, heart rate of 86 , blood pressure of $114 / 60$, respiratory rate 20 and oxygen saturation of $95-99 \%$ on room air. Her blood glucose was 557 (reference: 70 - $110 \mathrm{mg} / \mathrm{dL}$ ) on admission which corrected to 310 after receiving 20 units of regular insulin and $2 \mathrm{~L}$ of intravenous (IV) fluids in boluses. Lactic acid on admission was 2.6 (reference: 0.5 - $1.9 \mathrm{mmol} / \mathrm{L}$ ) which appropriately down-trended with fluid resuscitation. Her serum electrolytes were unremarkable with no evidence of an anion gap. Her complete blood count demonstrated no 
evidence of leukocytosis or leukopenia. Her urine analysis was notable for $3+$ blood, $2+$ glucose, $3+$ leukocyte esterase, seven red blood cells, few bacteria and no ketones. Her chest X-ray revealed a subtle, patchy, left-mid lung zone opacity similar to prior and thought to represent residual findings of known COVID-19 infection. On initial exam, she denied any specific focal symptoms including fevers, chills, dyspnea, chest pain, myalgias, or weakness. It should be mentioned that the patient had significant difficulty providing a cogent history or articulating any symptoms given her baseline cognitive impairment. Corroborating history provided by her group-home caretakers noted decline in physical mobility since her previous hospitalization; she was no longer able to transfer and sit in her wheelchair, which she had been able to do prior to her COVID-19 illness.

On day 2, nursing noted that patient's urine output had decreased. Daily lab work which was expanded to include our institutions screening and prognostic testing routinely used for COVID-19 patients returned with CK elevation of 16,843 (reference: 33 - $211 \mathrm{U} / \mathrm{L}$ ) with aspartate transaminase (AST) 425 (reference: 6 - $40 \mathrm{U} / \mathrm{L}$ ), alanine aminotransferase (ALT) 86 (reference: 10 - $49 \mathrm{U} / \mathrm{L}$ ) and creatinine 0.63 (reference: $0.6-1.5 \mathrm{mg} / \mathrm{dL}$ ). During her recent acute hospitalization for COVID-19 pneumonia, her CK ranged from 85 to 116 . There was no history of trauma reported by patient nor her facility.

Initial management of rhabdomyolysis included aggressive IV fluid rehydration with close monitoring of her CK, renal function and electrolytes. Her recently initiated statin was discontinued as a potential contributing factor. In further review of her medication list, chronic risperdal treatment for bipolar disorder was similarly discontinued though felt less likely given long term use. Neurology was consulted and electroencephalogram (EEG) was performed with no concern for seizure activity. CK peaked at 43,720 on day 4 at which time surgery was consulted to evaluate for possible compartment which was not felt to be likely. CK then began to downtrend.

On day 6, patient was noted to have developed anasarca with diffuse $2+$ pitting edema without evidence of respiratory compromise. This was subsequently treated with IV diuresis throughout the remainder of her hospitalization. Anasarca was thought to be due to aggressive fluid resuscitation in a patient with low albumin. IV fluids were completely discontinued on day 10 by which time her CK was 3,926. At the time of discharge, repeat nasal swab for COVID-19 was negative with CK down to 1,618. Her electrolytes remained stable through hospitalization and her liver function tests continued to downtrend concurrently with her CK. Renal function remained stable with a $\mathrm{Cr}$ of $0.4-0.6$ throughout. Blood glucose was stabilized on regimen of long acting and mealtime insulin. She was discharged to an inpatient rehabilitation facility.

\section{Discussion}

Often several "insults" upon muscle tissue are needed for the development of rhabdomyolysis [7]. In our patient, we suspect the etiology of rhabdomyolysis was multifactorial - a combination of prolonged immobility and physical deconditioning from COVID-19, exposure to a statin against a background of chronic neuroleptic medications, and potential viral-mediated myositis from SARS-CoV-2. While the pathophysiology of a SARS-CoV-2 mediated myositis is unclear, many viral infections such as influenza A and B have been known to cause rhabdomyolysis albeit typically in pediatric populations [7]. To our knowledge, there are three other case reports published in the recent months which describe rhabdomyolysis concurrently presenting with COVID-19 infection in adults [8-10]. In two of these cases [8,9], COVID-19 patients presented with an early manifestation of rhabdomyolysis with CK of 13,581 and CK $>42,670$ on admission, respectively, while Jin and Tong (2020) reported a delayed manifestation of rhabdomyolysis with CK of 11,842 on day 9 of admission. In two of the cases $[8,10]$, patients presented with pain and weakness in lower limbs, whereas in Zhang (2020), the patient reported diffuse myalgia. Our patient did not spontaneously voice myalgia or weakness albeit this may be confounded by her level of cognitive impairment.

This case presents a late-onset diagnosis of rhabdomyolysis in an adult patient 22 days after initial diagnosis of COVID-19 pneumonia. She was treated appropriately with aggressive IV fluids and was able to avoid complications including acute renal failure and compartment syndrome. Per review of literature, there is no standard protocol for duration and rate of fluid administration in rhabdomyolysis which remains an area of future research [7]. With our patient we also experienced a similar challenge as described by Suwanwongse et al (2020) in which aggressive fluid rehydration had to be balanced with concern for volume overload in a patient with recent admission for COVID-19-related hypoxia. Fluid stewardship becomes especially important in the subset of critically ill COVID-19 patients who develop acute respiratory distress syndrome (ARDS) as to not exacerbate pulmonary edema from aggressive fluid resuscitation [11]. Although our patient did not develop respiratory compromise, she ultimately needed to receive diuretic therapy to address volume overload from IV fluid rehydration.

Our case adds to small but emerging body of literature to suggest that rhabdomyolysis may be an associated complication of COVID-19 infection. As we see more patients readmitted to the hospital after initial recovery from COVID-19, we should continue to have a high clinical index of suspicion for potential complications of this novel virus, including rhabdomyolysis. As in this case presentation may be sub-clinical or atypical, so early recognition and management by clinicians is warranted as it could potentially avoid significant complications.

\section{Acknowledgments}

We would like to express our gratitude in being able to be involved in the care of this patient. We would also like to thank her legal guardian for providing informed consent on behalf of the patient so that we may share her case with the greater medical community. Finally, we would like to thank everyone involved in the care of this patient. 


\section{Financial Disclosures}

This publishing of this case report was sponsored by the corresponding author's annual resident education stipend subsidized by Beth Israel Deaconess Medical Center.

\section{Conflict of Interest}

None to declare.

\section{Informed Consent}

Informed consent for publication was provided by the patient's legal guardian.

\section{Author Contributions}

All authors contributed equally in terms of intellectual development of the case report, analysis of data and grammatical revision of the manuscript

\section{Data Availability}

The data supporting the findings of this study are available from the corresponding author upon reasonable request.

\section{References}

1. Cascella M, Rajnik M, Cuomo A, Dulebohn SC, Di
Napoli R. Features, evaluation and treatment coronavirus (COVID-19). In: StatPearls. Treasure Island (FL), 2020.

2. Zhai P, Ding Y, Wu X, Long J, Zhong Y, Li Y. The epidemiology, diagnosis and treatment of COVID-19. Int J Antimicrob Agents. 2020;55(5):105955.

3. Mao L, Jin H, Wang M, Hu Y, Chen S, He Q, Chang J, et al. Neurologic manifestations of hospitalized patients with coronavirus disease 2019 in Wuhan, China. JAMA Neurol. 2020.

4. Cipollaro L, Giordano L, Padulo J, Oliva F, Maffulli N. Musculoskeletal symptoms in SARS-CoV-2 (COVID-19) patients. J Orthop Surg Res. 2020;15(1):178.

5. Chavez LO, Leon M, Einav S, Varon J. Beyond muscle destruction: a systematic review of rhabdomyolysis for clinical practice. Crit Care. 2016;20(1):135.

6. Torres PA, Helmstetter JA, Kaye AM, Kaye AD. Rhabdomyolysis: pathogenesis, diagnosis, and treatment. Ochsner J. 2015;15(1):58-69.

7. Khan FY. Rhabdomyolysis: a review of the literature. Neth J Med. 2009;67(9):272-283.

8. Suwanwongse K, Shabarek N. Rhabdomyolysis as a presentation of 2019 novel coronavirus disease. Cureus. 2020;12(4): e7561.

9. Zhang Q, Shan KS, Minalyan A, O'Sullivan C, Nace T. A rare presentation of coronavirus disease 2019 (COVID-19) induced viral myositis with subsequent rhabdomyolysis. Cureus. 2020;12(5):e8074.

10. Jin M, Tong Q. Rhabdomyolysis as potential late complication associated with COVID-19. Emerg Infect Dis. 2020;26(7):1618-1620.

11. Kazory A, Ronco C, McCullough PA. SARS-CoV-2 (COVID-19) and intravascular volume management strategies in the critically ill. Proc (Bayl Univ Med Cent). 2020;0(0):1-6. 